Gut, 1974, 15, 515-520

\title{
Interpretation of fluctuation of transmural potential difference across the proximal small intestine
}

\author{
DAVID WINGATE, ROGER GREEN, JOHN SYMES, AND MARIANNE PILOT
}

From the Departments of Physiology and Experimental Surgery, London Hospital Medical College, London

SUMmARY The duodenal transmural potential difference (pd) has been studied in isolated vascularperfused preparations of canine stomach and duodenum. There was no quantitative correlation between the magnitude of the intraluminal pressure change and pd but fluctuations of pd in these preparations were related to duodenal slow wave activity.

The transmural potential difference across the wall of the proximal small intestine is a physiological phenomenon of increasing interest. Measurements of pd are a useful means of locating the precise site of the gastroduodenal junction (Andersson and Grossman, 1965). Recently, a technique for monitoring small intestinal pd in man has been described (Wingate, 1973) and changes in association with intestinal absorption have been observed (Wingate, Hayward, Johnson, Marczewskin, Petty, and Wilson, 1973.)

The small intestinal pd is of low magnitude (less than 10 millivolts), and measurement in vivo is hampered by the existence of fluctuations of the order of \pm 2 millivolts. Without a continuous recording system this introduces subjective error in noting voltage readings from a visual display. The question arises as to whether such fluctuations are no more than interference or whether they may provide useful information. This can only be answered when the origin of the fluctuation is identified: obvious possibilities include varying intraluminal pressure and intramural myoelectric activity. Preparations maintained in vitro by mucosal oxygenation, such as the everted sac, are not suitable for investigating this phenomenon, since they do not display spontaneous pressure changes or myoelectrical activity, nor do pd measurements in such preparations exhibit fluctuation.

In this institution considerable experience has accumulated in the use of isolated vascular-perfused canine stomach and proximal intestine preparations (Ritchie and Hardcastle, 1973) in which propagated electrical activity and motility are preserved (Green Received for publication 11 April 1974. and Hardcastle, 1971). The system allows the continuous recording of the basic electrical rhythm from the stomach, with a frequency of about $5 / \mathrm{min}$ (Kelly, Code, and Elveback, 1969), and from the duodenum with a frequency of about 19/min (Hermon-Taylor and Code, 1971).

Atropine has been shown to abolish spike potentials, and the consequent intraluminal pressure changes in these isolated preparations (Green and Hardcastle, 1971). Therefore we used atropine and gastrointestinal hormones in this study to dissociate mechanical events and propagated electrical changes. The absence of respiratory excursions and myocardial activity in this system allows the relationship between pd, pressure, and myoelectrical change to be studied without the interference of changes arising outside the stomach and duodenum.

\section{Methods}

ANIMALS

The stomach and duodenum of seven adult greyhounds were removed under general anaesthesia and maintained at $37^{\circ} \mathrm{C}$ by vascular perfusion with oxygenated blood from the donor animal. The operative technique and experimental apparatus are fully described elsewhere (Green and Hardcastle, 1970; Ritchie and Hardcastle, 1973). Throughout the experiments, generally lasting about two hours, perfusion flow and pressure were monitored continuously, and arterial $\mathrm{PO}_{2}$ and $\mathrm{pH}$ measured at frequent intervals.

PHARMACOLOGICAL AGENTS

After electrical activity had been recorded for a 
basal period, $50 \mu \mathrm{g}$ of atropine was rapidly injected into the arterial supply of the preparation. In three experiments, before the administration of atropine, $100 \mu \mathrm{g}$ of pentagastrin (Peptavlon, ICI) or 0.5 units of purified cholecystokinin (Jorpes) was similarly administered as bolus arterial injections.

\section{MYOELECTRICAL ACTIVITY}

Myoelectrical activity was monitored through silversilver chloride electrodes sutured to the serosal surface of the stomach and duodenum. A total of six sites was used, illustrated in fig 1 , three each for the stomach and the duodenum. In each experiment, up to four sites were monitored simultaneously through pre-amplifiers connected to AC amplifier stages of an eight-channel Mingograf 81 ink-jet recorder (Elema-Schonander). Intraluminal pressure was recorded from an open-tip tube connected, through a transducer and amplifier, to one channel of the ink-jet recorder. The open-tip tube was sited so that the distal opening was adjacent to the open end of the luminal 'salt bridge' pd electrode.

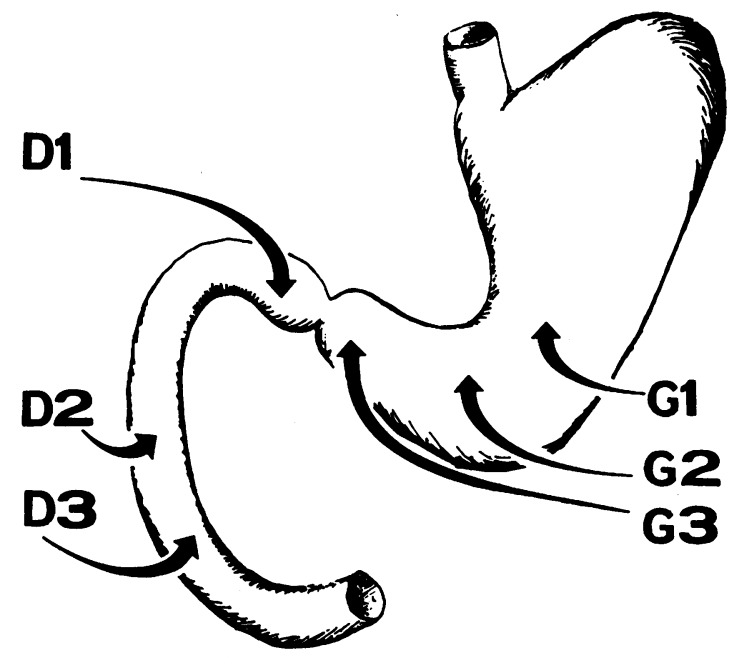

Fig 1 Sites used in this study for myoelectrical recording from the serosal surface of the stomach and duodenum.

TRANSMURAL PD

'Salt bridges', consisting of transparent polyvinyl chloride tubing filled with $2 \%$ agar-agar in $0.9 \%$ sodium chloride solution, were connected through standard calomel reference cells to the input of the measuring system. The distal tip of one electrode was placed on the serosal surface of the isolated organ, while the other was inserted in the lumen of the isolated duodenum through a large-bore cannula tied into the distal cut end of the intestine. The input from the calomel electrodes was connected to the input stage of an amplifier (type AD 55, Fenlow Electronics Ltd) with an input impedance of 100000 megohns, and subjected to a gain of 1000 . The amplified signal was divided between an appropriately calibrated digital voltmeter (type AD 2001, Analog Devices), with a measurement time of $250 \mathrm{msec}$, giving a direct read out to the nearest 0.1 millivolts on a three and a half digit Numitron display, and an attenuator connected to the ink-jet recorder. The pd measurement system was calibrated using a Weston standard cell connected through resistors of appropriate value to the amplifier input.

\section{Results}

The results are illustrated in figs 2-6, which are labelled according to a common convention. Myoelectrical records are labelled G1, G2, G3, D1, D2, or D3, according to the recording site as shown in figure 1. The intraluminal pressure record is marked ' $\mathrm{cm}$ ' with a vertical scale line which indicates the pressure in $\mathrm{cm}$ water, zero being atmospheric pressure. The pd recording is denoted by $\mathrm{mV}$ with an accompanying vertical scale line, indicating the electropositivity of the serosa in millivolts. The symbol $\mathrm{T}$ denotes a time marker showing one-second intervals.

In all preparations before the administration of pharmacological agents, fluctuations of pd and intraluminal pressure were always present, as illustrated in figures 2 and 3. The amplitude of fluctuations of pd was in the range of $\pm 1 \mathrm{mV}$ about the mean pd whereas the amplitude of pressure change was much more variable. Figures 2 and 3 clearly show that there is no relation between the amplitude of pressure change and the amplitude of pd change, although both fluctuate at similar frequencies.

At no point did there appear to be any relation between intraluminal pressure and sustained pd change of the magnitude and time course associated with active small intestinal absorption in vitro (Barry, Dikstein, Matthews, Smyth, and Wright, 1964) and in vivo (Wingate, Hayward, Johnson, Marcewski, Petty, and Wilson, 1973). In one experiment (but not in others) a bolus injection of cholecystokinin into the arterial circulation was followed by a pd rise of 2 millivolts, lasting about one minute, and associated with a considerable increase in myoelectrical activity and intraluminal pressure change; this sequence is illustrated in figure 4.

Atropine was used to dissociate myoelectrical and mechanical phenomena. Figure 5 shows a typical 


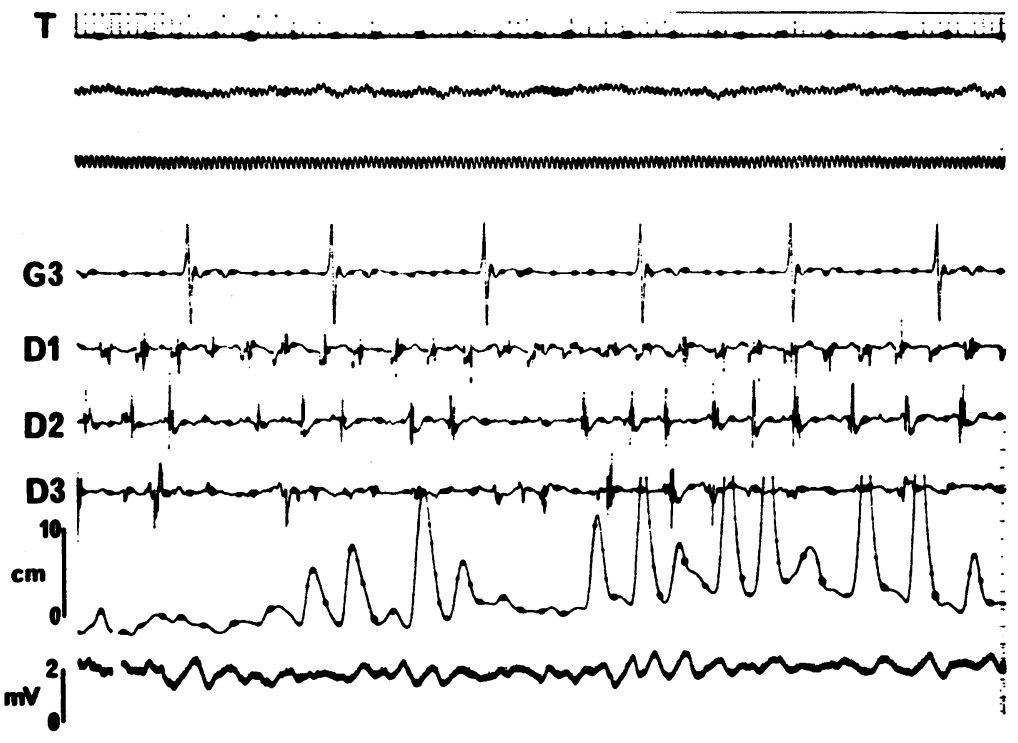

Fig 2 Simultaneous recording of $p d$ and intraluminal pressure in duodenum at D3 (see text for explanation of symbols).

result. Normal rhythms of myoelectrical, pressure, and pd change are seen before atropine (fig 5A); after atropine, in the same preparation, motility ceases with no change in intraluminal pressure, but the rhythm of myoelectrical and pd change continues at approximately 20 cycles per minute (fig 5B). The dissimilar waveforms of myoelectrical and pd change reflect the different electrical parameters of the recording systems, but the frequency is identical. The inference is that the pd fluctuation reflects propagated myoelectric activity.
This inference is supported by the absence of fluctuation in pd when myoelectrical activity is abolished. Physiological concentrations of gastrin enhance myoelectrical activity in this isolated preparation (Green and Hardcastle, 1971) as in man (Waterfall, Duthie, and Brown, 1973); with larger doses, a rapid increase in frequency of the basic duodenal electrical rhythm is followed by electrical standstill. Figure 6 compares duodenal pressure change, myoelectrical activity, and pd before (A) and following (B) arterial injection of $100 \mu \mathrm{g}$ of penta-

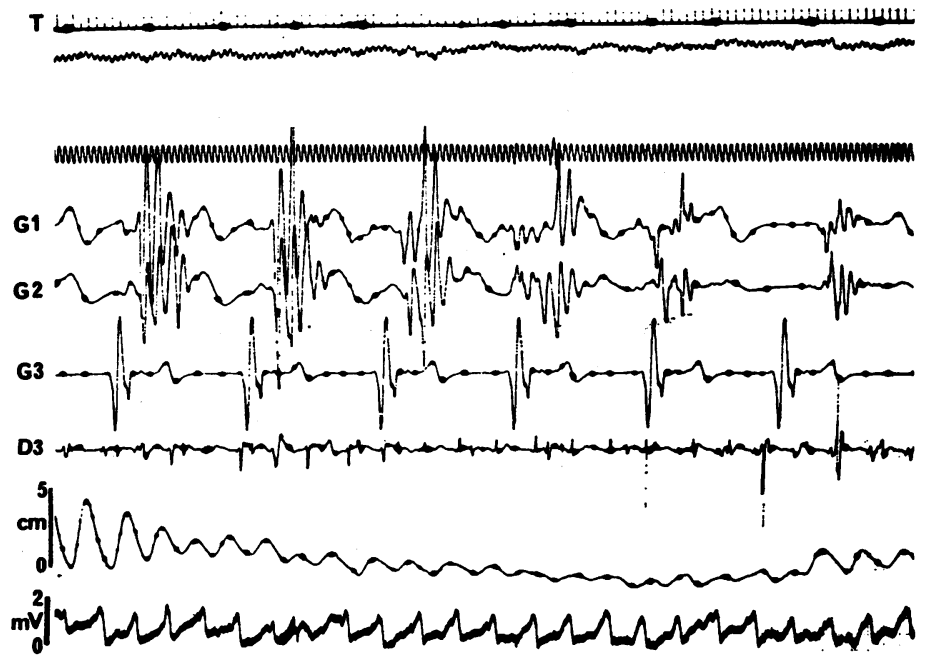

Fig 3 Simultaneous recording of $p d$ and intraluminal pressure in duodenum at D3 (see text for explanation of symbols). 
$\mathbf{T}$

: : ':

.

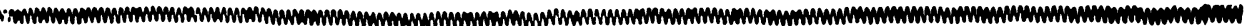

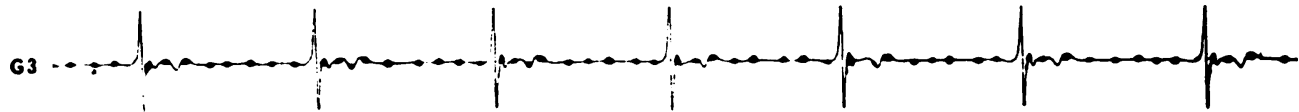

01 r

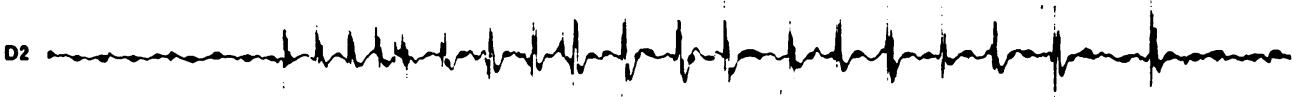

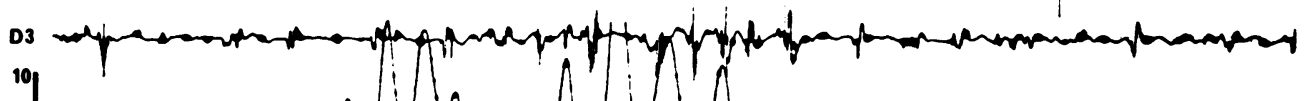

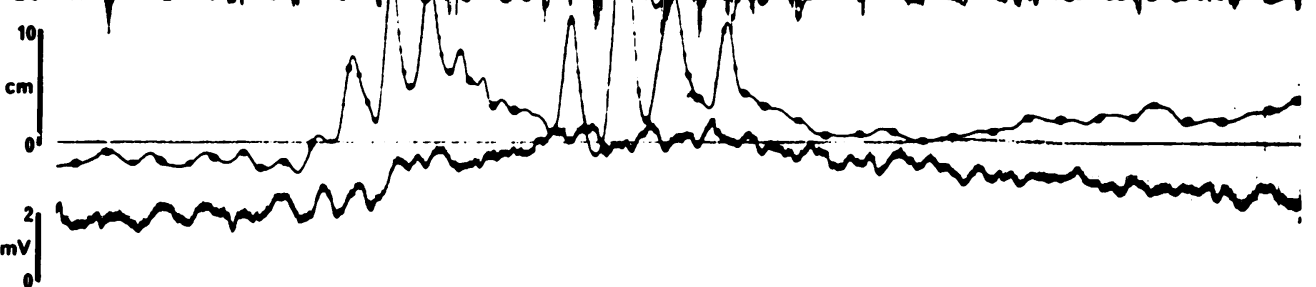

Fig 4 Simultaneous recording of $p d$ and intraluminal pressure in duodenum at D3. with record starting one minute after intraarterial injection of cholecystokinin/ pancreozymin (see text for explanation of symbols).

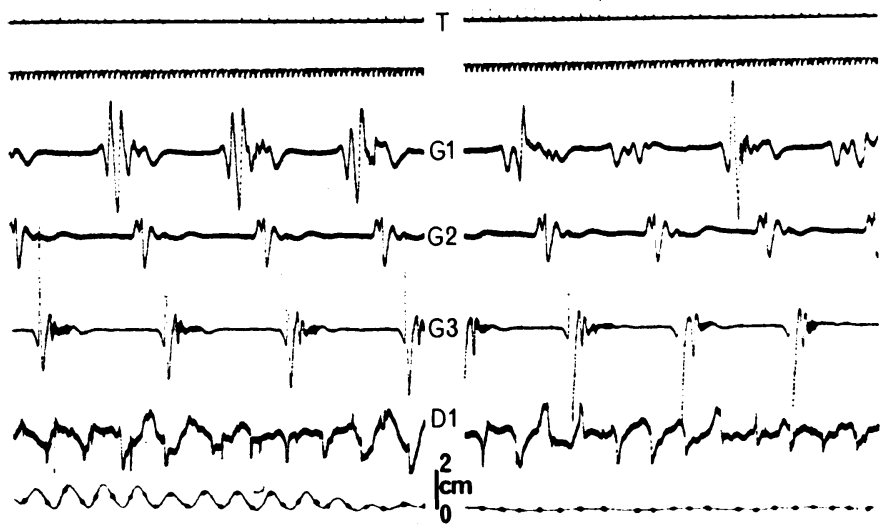

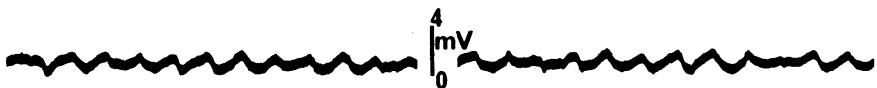

Fig 5 Simultaneous recording of $p d$ and intraluminal pressure in duodenum at $\mathrm{Dl}$ (A) before and (B) after administration of atropine (see text for explanation of symbols). 


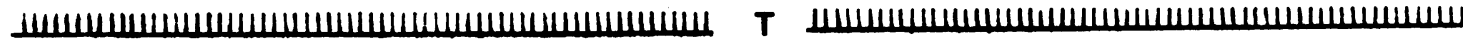
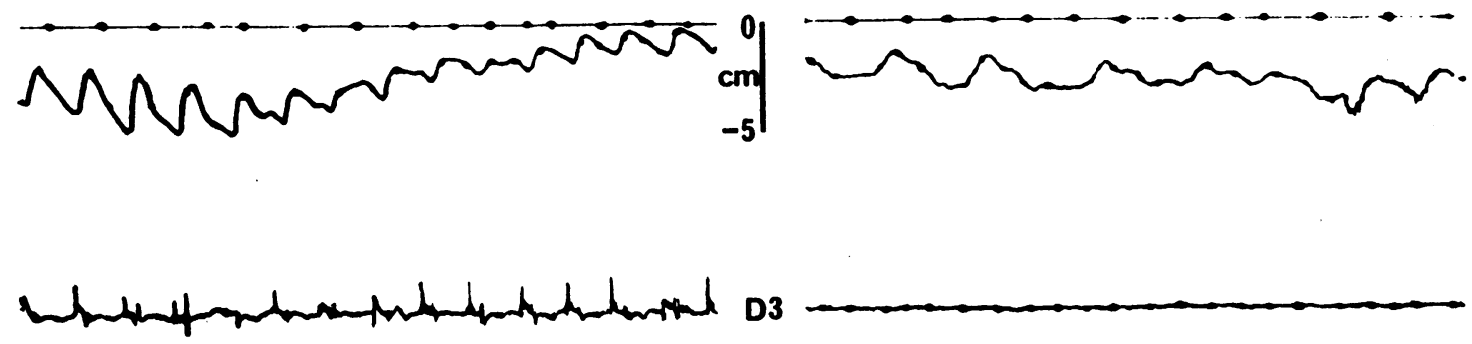

D3
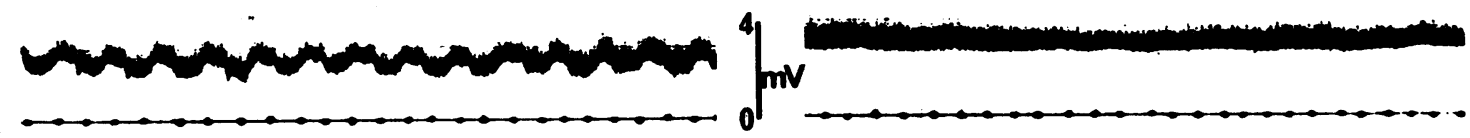

A

B

Fig 6 Simultaneous recording of pd and intraluminal pressure in duodenum at D3 (A) before and (B) after the administration of pentagastrin (see text for explanation of symbols).

gastrin. The effect of pentagastrin is to abolish the basic duodenal electrical rhythm; the pd record is now flat in spite of a rhythmical fluctuation in intraluminal pressure. The fluctuation in intraluminal pressure is altered from 20 cycles $/ \mathrm{min}$ to 5 cycles $/ \mathrm{min}$; gastric motility is preserved, and intragastric pressure change is transmitted to the lumen of the duodenum through an incompetent pyloric sphincter. The slow intraluminal pressure fluctuation is not reflected in the pd record. Rhythmic duodenal myoelectrical activity reduced in rate and amplitude, returned five to 10 minutes after pentagastrin administration, and a parallel return of pd fluctuation similarly reduced in rate and amplitude was observed.

\section{Discussion}

Barry (1967) was able, even some years ago, to cite a considerable volume of work on pd change in small intestine in vitro. There is a contrasting lack of evidence, even now, on pd changes in small intestine in situ, in which normal myoelectrical and mechanical change is preserved. Considering the difficulties of measuring small intestinal pd safely with existing equipment, this situation is not surprising.

The starting point of this investigation was a study of small intestinal pd change in man (Wingate et al, 1973) using newly developed high impedance measuring apparatus (Wingate, 1973) during which considerable fluctuation of the pd within narrow limits $( \pm 2 \mathrm{mV}$ of the mean pd) was observed. While it would not be difficult to devise electronic apparatus to filter such fluctuations, we felt that the phenomenon deserved investigation before being rejected. Our first speculation was that it might merely represent instability of the measuring circuit, possibly due to a varying spatial relationship of the mucosal probe to the mucosa. Secondly, we could not ignore the possibility that the fluctuation was following intraluminal pressure change. Since the intestinal mucosa, across which the pd is generated, acts as a complex ohmic resistor (Schultz, 1973), it is possible that mechanical distortion of the mucosa will alter tissue conductance and resistance. In vitro, it is known that tissue resistance does not vary during active absorption of glucose (Grady, Madoff, Duhamel, Chalmers, and Moore, 1967) or amino acids (Cotterell and Kohn, 1970) although conductance may vary if the ambient ions are altered (Schultz, Curran, and Wright, 1967).

In the event, our results do not support either of our two suppositions. The fluctuation appears to be not random but related in time to the basic electrical rhythm in the intestinal wall. Since our electrodes are measuring across the muscle layers as well as across the mucosa, and since the amplitude of the propagated potentials in the small intestine, approximately $2 \mathrm{mV}$ (Hermon-Taylor and Code, 1971), is similar in magnitude to the mucosal potential, this is a logical explanation of our findings. We interpret our results as showing that there are two components to the recorded pd change: the mucosal pd which 
changes relatively slowly, and a submucosal pd which reflects the propagated slow wave, changing relatively fast.

The inference from our findings is that small intestinal transmural pd measurement in vivo may produce information on the propagated electrical activity of the intestine as well as on transmucosal dc potential. Testing this speculation in man imposes further problems of clinical convenience and safety (Shaw and Monk, 1973), on which further studies are in progress.

\section{References}

Andersson, S., and Grossman, M. I. (1965). Profile of pH, pressure and potential difference at gastroduodenal junction in man. Gastroenterology, 49, 364-371.

Barry, R. J. C. (1967). Electrical changes in relation to transport. Brit. med. Bull., 23, 266-269.

Barry, R. J. C., Dikstein, S., Matthews, J., Smyth, D. H., and Wright, E. M. (1964). Electrical potentials associated with intestinal sugar transfer. J. Physiol. (Lond.), 171, 316-338.

Cotterell, D., and Kohn, P. G. (1970). Variation in tissue resistance in rat small intestine: Its relationship to observed potential changes. Biochim. biophys. Acta (Amst.), 203, 179-181.
Grady, G. F., Madoff, M. A., Duhamel, R. C., Moore, E. W., and Chalmers, T. C. (1967). Sodium transport by human ileum in vitro and its response to cholera enterotoxin. Gastroenterology. $53,737-744$.

Green, W. E. R., and Hardcastle, J. D. (1971). The myoelectrical activity of the isolated perfused canine stomach. J. Physiol. (Lond.), 222, 41P.

Hermon-Taylor, J., and Code, C. F. (1971). Localization of the duodenal pacemaker and its role in the organization of duodenal myoelectric activity. Gut, 12, 40-47.

Kelly, K. A., Code, C. F., and Elveback, L. R. (1969). Patterns of canine gastric electrical activity. Amer. J. Physiol., 217, 461-470.

Ritchie, H. D., and Hardcastle, J. D. (1973). Isolated Organ Perfusion. Crosby Lockwood Staples, London.

Schultz, S. G. (1972). Electrical potential difference and electromotive forces in epithelial tissues. J. gen. Physiol., 59, 794-798.

Schultz, S. G., Curran, P. F., and Wright, E. M. (1967). Interpretation of hexose-dependent electrical potential difference in the small intestine. Nature (Lond.), 214, 509-510.

Shaw, A., and Monk, I. B. (1973). Ingress of saline into electrical equipment. Lancet, 2, 794-795.

Waterfall, W. E., Duthie, H. L., and Brown, B. H. (1973). The electrical and motor actions of gastrointestinal hormones on the duodenum in man. Gut, 14, 689-696.

Wingate, D. L. (1973). A miniature digital millivoltmeter for measuring intestinal transmural electric potential difference. Gut, $14,399-401$.

Wingate, D. L., Hayward, M. G., Johnson, C. M., Marczewski, A. G Petty, R. G., and Wilson, E. J. (1973). Physiological change. in human transjejunal potential difference. Scand. J. Gastroent. $8,473-478$. 